\title{
Optimizing Workflows in Correlative Light and Electron Microscopy.
}

\author{
Kirk Czymmek ${ }^{1}$, Jeff Caplan² ${ }^{2}$ Cherish Warner ${ }^{3}$, Janine Sherrier ${ }^{3}$ and Alexandra Elli ${ }^{4}$ \\ 1. Carl Zeiss Microcopy, LLC, One Zeiss Drive, Thornwood, NY 10594, USA \\ 2. 15 Innovation Way, Delaware Biotechnology Institute, University of Delaware, Newark DE 19711, \\ USA \\ 3. 15 Innovation Way, Delaware Biotechnology Institute, Department of Plant and Soil Sciences, \\ University of Delaware, Newark DE 19711, USA \\ 4. Carl Zeiss Microscopy, Carl-Zeiss-Strasse 22, 73447 Oberkochen, Germany
}

Correlative microscopy allows researchers to look at the same exact structure of interest using typically separate, but powerful microscopy platforms commonly including light and electron microscopy [1]. By combining the unique imaging modalities to study singular biological events, more detailed investigations of structure-function relationships can be conducted that greatly facilitate our understanding of cellular phenomenon. With photon-based microscopy, rare structures or targeted phenomenon in cells and tissues can be readily located and imaged due to the large field-of-view and ease of sample screening. Conventional light (resolution $\sim 0.2 \mu \mathrm{m}$ ) and super-resolution (resolution up to $\sim 20 \mathrm{~nm}$ ) microscopies are well-positioned to benefit from the high resolution subcellular or ultrastructural details inherent to electron microscopy for correlating the spatial distribution of macromolecules at nanoscale resolutions.

In one example provided here, we used correlative microscopy combining immunofluorescence and light microscopy on epoxy resin sections followed by field emission scanning electron microscopy to provide key structural data at high-resolution over large areas (Fig 1A-D.). This work sought to understand the spatial symbiotic relationships between nitrogen-fixing bacteria rhizobia and the host legume plant, Medicago truncatula in root nodules. Samples were fixed with 1\% glutaraldehyde and 4\% paraformaldehyde in 100mM PIPES and then post-fixed in 1\% osmium tetroxide (aq). Subsequently, nodules were dehydrated in acetone, infiltrated and embedded in an Epon-Araldite epoxy resin and serial sections acquired and dried down on 22 x 22mm indium tin oxide coated-coverslips with fiducials marks. Sections were further processed for immunofluorescence using a callose primary antibody and AlexaFluor ${ }^{\circledR} 647$ secondary antibody which labeled plasmodesmata traversing the cell walls (Fig 1 A,B \& D). Light microscopy was performed using Shuttle \& Find on a ZEISS AxioImager and then samples were stained with lead citrate and uranyl acetate and then relocated and imaged with a backscatter detector on a ZEISS SIGMA HD VP FESEM at $8 \mathrm{kV}$ and at $10 \mathrm{~nm}$ pixel resolution.

This presentation will focus on a variety of contemporary methods, probes and workflow for correlative light and electron microscopy (CLEM) and will demonstrate with real world examples of animals, plants and microbes, strategies to facilitate relocation of microscopic data for correlative experiments including automation and 3-D approaches [2]. 


\section{References:}

[1] J. Caplan, M. Niethammer, R. Taylor and K Czymmek, Current Opinion in Structural Biology 21 (2011), p. 686-693.

[2] The authors acknowledge funding from NSF-IOS-1127155 to D.J.S. and NSF-IOS-0923668 to D.J.S
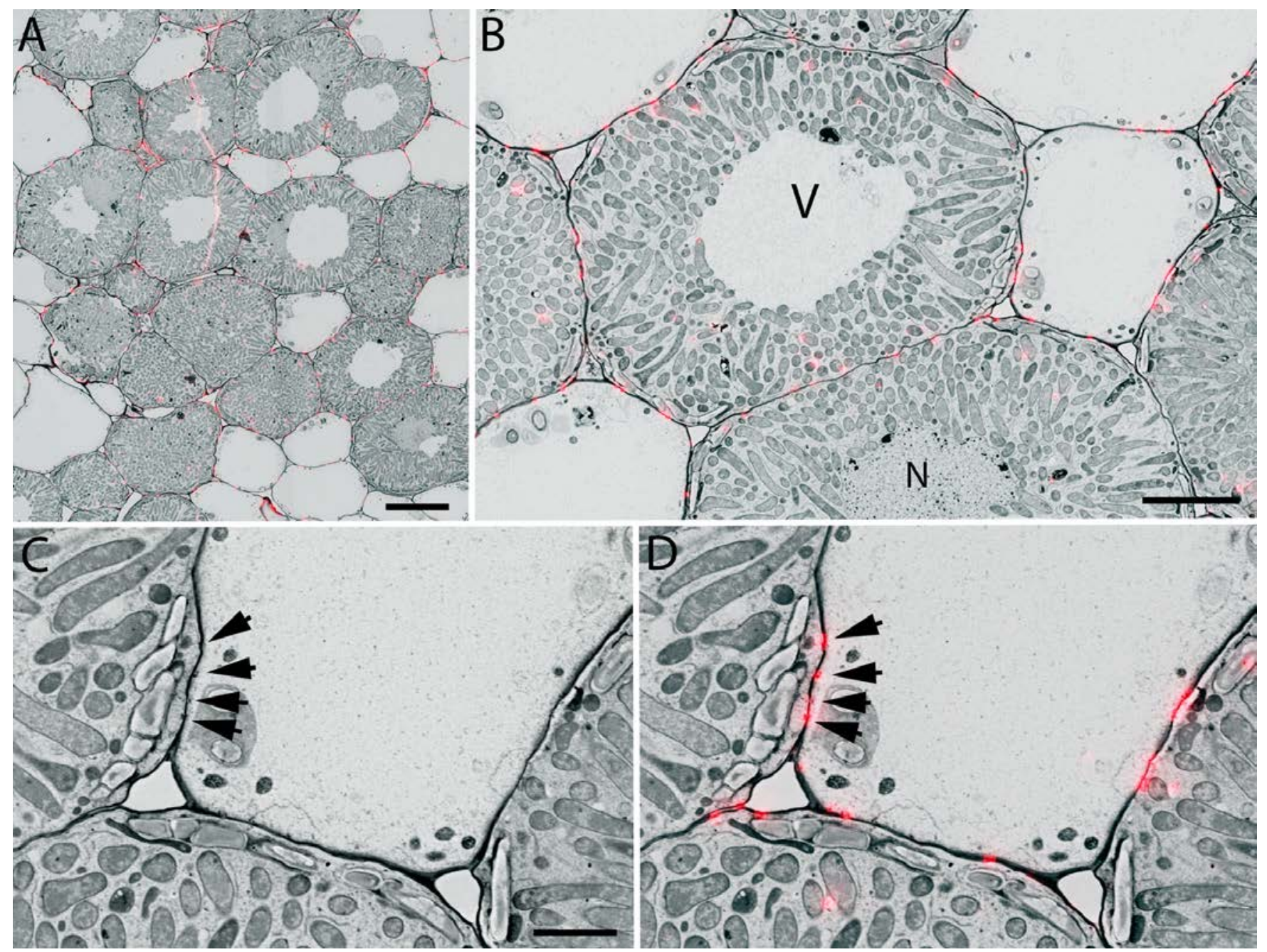

Figure 1. CLEM of nitrogen-fixing bacteria rhizobia and the host legume plant, Medicago truncatula in root nodules. 1A. Low magnification overview of the section showed immunolabeled callose (red) localized along the cell walls. Scale bar $=25 \mathrm{um}$. 1B. Closer inspection of the sample dataset showed abundant rhizobia within individual plant cells and a large central vacuole (V) and small puncta (red) localized along the cell walls. $(\mathrm{N})$ nucleus. Scale bar $=10 \mathrm{um}$. 1C. At higher magnification, small channels representing plasmodesmata could be observed traversing the cell wall (arrows) in the FESEM image. Scale bar $=3 \mathrm{um}$. 1D. A correlative overlay of the fluorescence channel (red) localizing to callose confirmed the antibody localized to plasmodesmata (arrows). Scale bar = 3um. 